\title{
SOME INTEGRAL INEQUALITIES
}

RICHARD P. GOSSELIN ${ }^{1}$

1. The purpose of this paper is to present a general integral inequality concerning subadditive functions and to make applications of this inequality. The applications pertain to relations among integrals involving first and second differences of $L^{p}$ functions. The finiteness of some of the integrals is connected with generalized Lipschitz conditions and with the existence of fractional derivatives. These facts are exploited to obtain both new and known theorems. Finally we show that in some cases the finiteness of the integral is not affected by interchanging the first and second differences of the function.

We say the positive measurable function $\phi$ is subadditive on the interval $(0, A), 0<A \leqq \infty$, if $\phi(u+v) \leqq \phi(u)+\phi(v)$ where $u, v$, and $u+v$ all belong to $(0, A)$. The first theorem states that for subadditive functions the $L^{p}$ norm, $p \geqq 1$, of $\phi(u) / u^{\alpha}$ with respect to the infinite measure $d u / u$ does not exceed a constant multiple of the $L$ norm of this function with respect to the same measure. Here $\alpha$ is any real number.

TheOREM 1. Let $\phi(u)$ be positive, measurable, and subadditive on $(0, A)$.

(i) Let $p \geqq 1$, and let $\alpha$ be any real number. There exists $C_{\alpha, p}$ depending only on its subscripts such that

$$
\left(\int_{0}^{A} \frac{\phi^{p}(u)}{u^{1+p \alpha}} d u\right)^{1 / p} \leqq C_{\alpha, p} \int_{0}^{A} \frac{\phi(u)}{u^{1+\alpha}} d u .
$$

(ii) If either integral above is finite, then there exists a constant $C$ depending on $\phi, p$, and $\alpha$ but not on $u$ such that $\phi(u) \leqq C u^{\alpha}$ for $u$ in $(0, A)$.

A very special case of this inequality is known: viz. when $\phi$ is decreasing and $\alpha=-1[2$, p. 39].

Let $M$ denote the value of the integral on the right in (1). $M$ may be assumed finite and strictly positive. Let $E$ denote the set of points $u$ such that $\phi(u)>M u^{\alpha} /(\log 4 / 3)$, and let $G$ be the complement of $E$. Then

Presented to the Society, March 9, 1961; received by the editors March 13, 1961 and, in revised form, May 7, 1961.

${ }^{1}$ This research was supported by the United States Air Force through the Air Force Office of Scientific Research of Air Research and Development Command, under Contract No. AF 49(638)-608. 


$$
\int_{E} \frac{1}{u} d u<\frac{\log (4 / 3)}{M} \int_{0}^{A} \frac{\phi(u)}{u^{1+\alpha}} d u=\log (4 / 3) .
$$

We assert that for every $u$ in $(0, A)$ there exists $v$ in $(u / 3,2 u / 3)$ such that $v$ belongs to $G$ and such that $w=u-v$ belongs to $G$. If this were not true, say for $u_{0}$, then $\left(u_{0} / 3,2 u_{0} / 3\right)=E_{0} \cup E_{1}$ where $E_{0}$ $=E \cap\left(u_{0} / 3,2 u_{0} / 3\right)$ and $E_{1}$ is the set of points of the form $v=u_{0}-w$ where $w$ belongs to $E_{0}$. Since $E_{0}$ and $E_{1}$ are reflections of each other through the point $u_{0} / 2$, they have the same measure, $\left|E_{0}\right|$. Thus $\left|E_{0}\right| \geqq u_{0} / 6$, and

$$
\log (4 / 3)=\int_{u_{0} / 2}^{2 u_{0} / 3} \frac{1}{u} d u \leqq \int_{E_{0}} \frac{1}{u} d u \leqq \int_{E} \frac{1}{u} d u<\log (4 / 3)
$$

by (2). This contradiction proves our assertion. Thus

$$
\begin{aligned}
\phi(u) & =\phi(v+w) \leqq \phi(v)+\phi(w) \\
& \leqq \frac{M}{\log (4 / 3)}\left(v^{\alpha}+w^{\alpha}\right) ; \quad v, w \text { in } G \cap(u / 3,2 u / 3) .
\end{aligned}
$$

If $\alpha \geqq 0, v^{\alpha}+w^{\alpha} \leqq 2 u^{\alpha}$. If $\alpha<0, v^{\alpha}+w^{\alpha} \leqq 2 u^{\alpha} / 3^{\alpha}$. Hence

$$
\phi(u) \leqq C_{\alpha} M u^{\alpha} / \log (4 / 3)
$$

and so

$$
\frac{\phi^{p}(u)}{u^{1+p \alpha}} \leqq C_{\alpha, p} M^{p-1} \frac{\phi(u)}{u^{1+\alpha}} .
$$

Integration over $(0, A)$ completes the proof of (i). (3) shows that $\phi(u) \leqq C \iota^{\alpha}$ if the right side of (1) is finite. If only the left side of (1) is finite, then the same proof holds except that $M$ must be replaced by the value of the corresponding integral. The constant can be improved somewhat by modification of the set $E_{0}$. In the case $\alpha \geqq 0$, it is bounded in $\alpha$ for each $p$.

It is a fact of some importance for applications that the theorem is vacuous for $\alpha \geqq 1$.

Theorem 2. Let $\phi$ be positive, measurable, and subadditive, and let $\int_{0}^{A} \phi^{p}(u) / u^{1+p \alpha} d u<\infty$ for some $p>0$ and $\alpha \geqq 1$. Then $\phi$ is identically zero.

It is enough to consider the case $\alpha=1$. We may take $A$ to be $\infty$ since $\phi$ may always be defined as 0 to the right of $A$. This does not affect the subadditivity property nor the finiteness of the above integral. If $\phi$ is not equivalent to 0 , there exists $B, 0<B<\infty$, such that 


$$
\int_{0}^{B} \frac{\phi^{p}(u)}{u^{1+p}} d u<\int_{0}^{\infty} \frac{\phi^{p}(u)}{u^{1+p}} d u
$$

Let $N$ be a large positive integer, and let $u=N v$. Since $\phi^{p}(N v)$ $\leqq N^{p} \phi^{p}(v)$,

$$
\int_{0}^{N B} \frac{\phi^{p}(u)}{u^{1+p}} d u \leqq \int_{0}^{B} \frac{\phi^{p}(v)}{v^{1+p}} d v .
$$

Now let $N$ approach $\infty$. The resulting contradiction shows that $\phi$ is equivalent to 0 , and it is not hard to see from this that it is identically 0 .

We mention briefly some variants of Theorem 1 with $\phi$ positive, measurable, and subadditive as before. Let $0 \leqq \alpha$ and $0<p<1$. Then

$$
\int_{0}^{A} \frac{\phi(u)}{u^{1+\alpha}} d u \leqq C_{p}\left(\int_{0}^{A} \frac{\phi^{p}(u)}{u^{1+p \alpha}} d u\right)^{1 / p} .
$$

Let $A$ be finite, and let $\alpha \geqq 0, \gamma>1, p \geqq 1$. Then

$$
\left(\int_{0}^{A} \frac{\phi^{p}(u)}{u^{\gamma+p \alpha}} d u\right)^{1 / p} \leqq C(p, \gamma, A) \int_{0}^{A} \frac{\phi(u)}{u^{\gamma+\alpha}} d u .
$$

The proofs of both inequalities follow the lines already established. It is also easy to see that (1) remains valid if we replace $1 / u^{\alpha}$ in both integrands by any positive, decreasing function.

2. Let $f$ be periodic of period $2 \pi$. For purposes of applying Theorem 1 , our chief concern will be with the function

$$
\phi_{r}(u ; f)=\left(\int_{0}^{2 \pi}|f(x+u)-f(x)|^{r} d x\right)^{1 / r}, \quad r \geqq 1,
$$

which is subadditive in view of Minkowski's inequality and the periodicity of $f$. Thus the statement of Theorem 1 (i) in the case $r=p \geqq 1, \alpha \geqq 0$, is

$$
\begin{aligned}
& \left(\int_{0}^{2 \pi} \int_{0}^{2 \pi} \frac{|f(x+u)-f(x)|^{p}}{u^{1+p \alpha}} d u d x\right)^{1 / p} \\
& \quad \leqq C_{p} \int_{0}^{2 \pi} \frac{d u}{u^{1+\alpha}}\left(\int_{0}^{2 \pi}|f(x+u)-f(x)|^{p} d x\right)^{1 / p} .
\end{aligned}
$$

As noted previously, if $\alpha \geqq 0$, we may take the constant independent of $\alpha$.

If $\alpha \geqq 1$, and if the left side of (4) is finite, then according to Theorem $2, \phi_{p}(u ; f)$ is identically 0 . This implies that $f$ is equivalent to a 
constant, a fact which is an integral analogue of an elementary result for functions satisfying an ordinary Lipschitz condition.

There are examples of functions for which the left side of (4) is finite while the right side is infinite. We define an $L^{2}$ function by its Fourier coefficients. Let

$$
f(x) \sim \sum_{n=2}^{\infty} c_{n} e^{i n x}, \quad c_{n}^{2}=\frac{1}{n^{1+2 \alpha}(\log n)^{\gamma}}, \quad 0<\alpha<1,1<\gamma \leqq 2 .
$$

The finiteness of the left side of (4) with $p=2$ is equivalent to the convergence of the series $\sum_{n=2}^{\infty} 1 / n(\log n)^{\gamma}$. For the integral on the right, it is convenient to split up the $u$-interval of integration into subintervals $\left(\pi / 2^{k}, \pi / 2^{k-1}\right), k=0,1, \cdots$. An easy calculation then shows that the divergence of the integral follows from the divergence of the series $\sum_{k=1}^{\infty} k^{-\gamma / 2}$.

Let $\Delta^{2} f(x, u)$ be the second symmetric difference of $f$, i.e., $\Delta^{2} f(x, u)$ $=f(x+u)+f(x-u)-2 f(x)$, and let

$$
\psi_{r}(u ; f)=\left(\int_{0}^{2 \pi}\left|\Delta^{2} f(x, u)\right|^{r} d x\right)^{1 / r}, \quad r \geqq 1 .
$$

Since

$$
\begin{aligned}
\Delta^{2} f(x, u+v)= & \Delta^{2} f(x+u, v)+\Delta^{2} f(x-u, v)+2 \Delta^{2} f(x, u) \\
& -\Delta^{2} f(x,|u-v|),
\end{aligned}
$$

then by Minkowski's inequality

$$
\psi_{r}(u+v ; f) \leqq 2 \psi_{r}(u ; f)+2 \psi_{r}(v ; f)+\psi_{r}(|u-v| ; f) .
$$

Using this inequality along with the techniques already established, we may show

$$
\left(\int_{0}^{2 \pi} \frac{\psi_{p}^{p}(u ; f)}{u^{1+p \alpha}} d u\right)^{1 / p} \leqq C_{p} \int_{0}^{2 \pi} \frac{\psi_{p}(u ; f)}{u^{1+\alpha}} d u, \quad p \geqq 1, \alpha \geqq 0 .
$$

This result has pertinence to a theorem of Offord [3].

3. The finiteness of the integrals in (4) involves in some cases the existence of fractional derivatives. Let $f^{(\alpha)}$ denote the fractional derivative of $f$ of order $\alpha$. The following inequalities are meant to imply the existence of fractional derivatives in appropriate circumstances and represent a sharpened form of (4).

Theorem 3. (i) Let $0<\alpha<1,1<p \leqq 2$. Then 


$$
\begin{aligned}
\left(\int_{0}^{2 \pi}\left|f^{(\alpha)}(x)\right| p d x\right)^{1 / p} & \leqq A_{p, \alpha}\left(\int_{0}^{2 \pi} \frac{\phi_{p}^{p}(u ; f)}{u^{1+p \alpha}} d u\right)^{1 / p} \\
& \leqq B_{p, \alpha} \int_{0}^{2 \pi} \frac{\phi_{p}(u ; f)}{u^{1+\alpha}} d u
\end{aligned}
$$

(ii) Let $0<\alpha<1,2 \leqq q$. Then

$$
\begin{aligned}
\left(\int_{0}^{2 \pi} \frac{\phi_{q}^{q}(u ; f)}{u^{1+q \alpha}} d u\right)^{1 / q} & \leqq C_{q, \alpha}\left(\int_{0}^{2 \pi}\left|f^{(\alpha)}(x)\right|^{q} d x\right)^{1 / q} \\
& \leqq B_{q, \alpha} \int_{0}^{2 \pi} \frac{\phi_{q}(u ; f)}{u^{1+\alpha}} d u
\end{aligned}
$$

It is convenient at this point to substitute $f(x+u)-f(x-u)$ for $f(x+u)-f(x)$ in the definition of $\phi_{p}(u ; f)$. This will not affect the above inequalities except possibly for a change in the constants. The first inequality in (i) is due to Hirschman [1], and the second is simply a special case of (4). The first inequality in (ii) is due to Offord [3], who states it using the second symmetric difference of $f$; but his proof is equally valid in this case. For the second inequality in (ii), we use the following due to Hirschman [1, p. 545]:

$$
\left(\int_{0}^{2 \pi}\left|f^{(\alpha)}(x)\right|^{q} d x\right)^{2 / q} \leqq B_{q, \alpha} \int_{0}^{2 \pi} \frac{\phi_{q}^{2}(u ; f)}{u^{1+2 \alpha}} d u .
$$

There is a misprint of the statement of this in [1] which accounts for a reversal of the inequality. Now it is enough to apply Theorem 1 to $\phi_{q}$ with $p=2$.

The proofs in [1] are rather complicated, and we now give a somewhat simplified proof of the first inequality in (i), which however still involves complex methods indirectly through the use of a theorem of Littlewood and Paley. Let $\sum c_{n} e^{i n x}$ be the Fourier series of $f$. Throughout the discussion of fractional derivatives, it is assumed that $c_{0}=0$. We may also assume that $c_{n}=0$ if $n<0$ (cf. [4]). The Fourier series of $f(x+u)-f(x-u)$ is then $2 i \sum_{n=1}^{\infty} c_{n} \sin n u e^{i n x}$. Let

$$
f_{k}(x)=\sum_{n=2^{k}}^{2^{k+1}-1} c_{n} e^{i n x}, \quad f_{k}^{(\alpha)}(x)=\sum_{n=2^{k}}^{2^{k+1}-1} n^{\alpha} c_{n} e^{i n x}, \quad k=0,1, \cdots
$$

Let $\pi / 2^{k+3} \leqq u \leqq \pi / 2^{k+2}$, and $2^{k} \leqq n<2^{k+1}$. Then $(\sin n u)^{-1}$ is monotone and bounded by $(\sin \pi / 8)^{-1}$ for fixed $u$ in the given range as $n$ increases from $2^{k}$ to $2^{k+1}$. Using the fact that the $L^{p}$ norm of a section 
of a Fourier series of a function does not exceed a constant multiple of the $L^{p}$ norm of the function, we have

$$
\begin{aligned}
\int_{0}^{2 \pi}\left|f_{k}(x)\right| p d x & \leqq A_{p} \int_{0}^{2 \pi}\left|\sum_{n=2^{k}}^{2^{k+1}-1} c_{n} \sin n u e^{i n x}\right|^{p} d x \\
& \leqq B_{p} \int_{0}^{2 \pi}|f(x+u)-f(x-u)|^{p} d x
\end{aligned}
$$

if $\pi / 2^{k+3} \leqq u \leqq \pi / 2^{k+2}$. We multiply the extreme terms of the above inequality by $1 / u^{1+p \alpha}$ and integrate over the given $u$ range to obtain

$$
\begin{aligned}
2^{k p \alpha} \int_{0}^{2 \pi}\left|f_{k}(x)\right|^{p} d x & \\
& \leqq C_{p, \alpha} \int_{\pi / 2^{k+3}}^{\pi / 2^{k+2}} \frac{d u}{u^{1+p \alpha}} \int_{0}^{2 \pi}|f(x+u)-f(x-u)|^{p} d x .
\end{aligned}
$$

The transformation from $2^{k \alpha} f_{k}(x)$ to $f_{k}^{(\alpha)}(x)$ is clearly bounded in $L^{p}$ so that summing over $k$ gives

$$
\sum_{k=0}^{\infty} \int_{0}^{2 \pi}\left|f_{k}^{(\alpha)}(x)\right|^{p} d x \leqq D_{p, \alpha} \int_{0}^{2 \pi} \frac{d u}{u^{1+p \alpha}} \int_{0}^{2 \pi}|f(x+u)-f(x-u)|^{p} d x .
$$

Let $f^{(\alpha)}(x)$ be the function defined by $\sum_{n \geq 1} n^{\alpha} c_{n} e^{i n x}$. By the theorem of Littlewood and Paley [4, p. 233],

$$
\int_{0}^{2 \pi}\left|f^{(\alpha)}(x)\right|^{p} d x \leqq E_{p} \sum_{k=0}^{\infty} \int_{0}^{2 \pi}\left|f_{k}^{(\alpha)}(x)\right|^{p} d x .
$$

Since $f^{(\alpha)}$ is, apart from a complex constant, the $\alpha$ th derivative of $f$, the proof is complete.

4. The point of our last theorem is that in some cases we may substitute the first difference of the function for the second, symmetric difference without affecting the finiteness of the integral involved. Let $\Delta f(x, u)=f(x+u)-f(x)$.

Theorem 4. Let $0<\alpha<1, p \geqq 1$. Let the periodic function $f$ belong to $L^{p}$. Then

$$
\begin{aligned}
\int_{0}^{2 \pi} \int_{0}^{2 \pi} \frac{|\Delta f(x, u)|^{p}}{u^{1+\alpha}} d u d x & \\
& \leqq C_{p, \alpha} \int_{0}^{2 \pi}|f(x)|^{p} d x+C_{p, \alpha} \int_{0}^{2 \pi} \int_{0}^{2 \pi} \frac{\left|\Delta^{2} f(x, u)\right|^{p}}{u^{1+\alpha}} d u d x
\end{aligned}
$$

Since the proof is an adaptation of a classical argument, we may be 
brief. Systematic use of the relation $\Delta f(x, u)-2 \Delta f(x, u / 2)$ $=\Delta^{2} f(x+u / 2, u / 2)$ leads to

$$
\Delta f\left(x, u / 2^{n}\right)=2^{-n} \Delta f(x, u)-2^{-n} \sum_{\nu=1}^{n} 2^{\nu-1} \Delta^{2} f\left(x+u / 2^{\nu}, u / 2^{\nu}\right) .
$$

Thus by Hölder's inequality (if $p>1$ ),

$$
\begin{aligned}
2^{-p / q}\left|\Delta f\left(x, u / 2^{n}\right)\right|^{p} & \\
& \leqq 2^{-n p}|\Delta f(x, u)|^{p}+2^{-n} \sum_{\nu=1}^{n} 2^{\nu-1}\left|\Delta^{2} f\left(x+u / 2^{v}, u / 2^{v}\right)\right|^{p} .
\end{aligned}
$$

We write

$$
\int_{0}^{2 \pi} \frac{|f(x, u)|^{p}}{u^{1+\alpha}} d u=\sum_{n=0}^{\infty} \int_{\pi / 2^{n}}^{\pi / 2^{n-1}} \frac{|\Delta f(x, u)|^{p}}{u^{1+\alpha}} d u
$$

and apply to each term of the series the above inequality. Thus

$$
\begin{aligned}
\int_{\pi / 2^{n}}^{\pi / 2^{n-1}} & \frac{|\Delta f(x, u)|^{p}}{u^{1+\alpha}} d u \\
& \leqq 2^{n \alpha} \int_{\pi}^{2 \pi} \frac{\left|\Delta f\left(x, v / 2^{n}\right)\right|^{p}}{v^{1+\alpha}} d v \leqq C_{p, \alpha} 2^{n(\alpha-p)} \int_{\pi}^{2 \pi} \frac{|\Delta f(x, u)|^{p}}{u^{1+\alpha}} d u \\
& +C_{p, \alpha} 2^{n(\alpha-1)} \sum_{\nu=1}^{n} 2^{\nu(1-\alpha)} \int_{\pi / 2^{\nu}}^{\pi / 2^{\nu-1}} \frac{\left|\Delta^{2} f(x+u, u)\right|^{p}}{u^{1+\alpha}} d u
\end{aligned}
$$

Since $\alpha<1 \leqq p$, summing over $n$ gives

$$
\begin{aligned}
\int_{0}^{2 \pi} \frac{|\Delta f(x, u)|^{p}}{u^{1+\alpha}} & d u \\
& \leqq C_{p, \alpha} \int_{0}^{2 \pi}|f(x)|^{p} d x+C_{p, \alpha} \int_{0}^{2 \pi} \frac{\left|\Delta^{2} f(x+u, u)\right|^{p}}{u^{1+\alpha}} d u .
\end{aligned}
$$

Integration with respect to $x$ and a change of variable in the second integral on the right complete the proof.

\section{REFERENCES}

1. I. I. Hirschman, Jr., Fractional integration, Amer. J. Math. 75 (1953), 531-546.

2. G. G. Lorentz, Some new functional spaces, Ann. of Math. 51 (1950), 37-55.

3. A. C. Offord, Approximation to functions by trigonometric polynomials. II, Fund. Math. 35 (1948), 259-270. 1959.

4. A. Zygmund, Trigonometric series, Vol. II, Cambridge Univ. Press, Cambridge,

University OF ConNecticut 